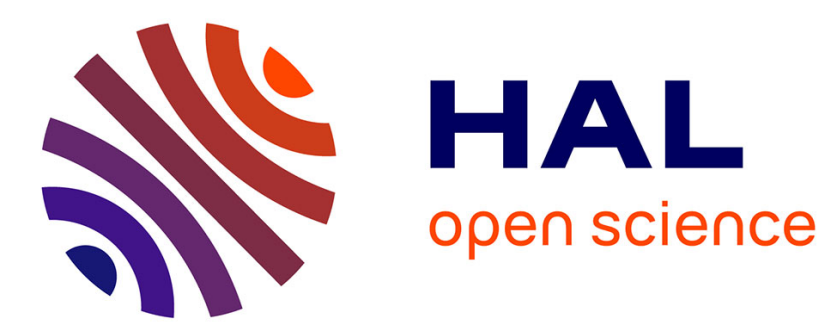

\title{
Mitoribosome assembly comes into view
}

\author{
Marie Sissler, Yaser Hashem
}

\section{To cite this version:}

Marie Sissler, Yaser Hashem. Mitoribosome assembly comes into view. Nature Structural and Molecular Biology, 2021, 28 (8), pp.631-633. 10.1038/s41594-021-00640-3 . hal-03365777

\section{HAL Id: hal-03365777 \\ https://hal.science/hal-03365777}

Submitted on 6 Oct 2021

HAL is a multi-disciplinary open access archive for the deposit and dissemination of scientific research documents, whether they are published or not. The documents may come from teaching and research institutions in France or abroad, or from public or private research centers.
L'archive ouverte pluridisciplinaire HAL, est destinée au dépôt et à la diffusion de documents scientifiques de niveau recherche, publiés ou non, émanant des établissements d'enseignement et de recherche français ou étrangers, des laboratoires publics ou privés. 


\title{
RIBOSOMES
}

\section{Mitoribosome assembly comes into view}

\author{
Marie Sissler ${ }^{1, *} \&$ Yaser Hashem ${ }^{1, *}$
}

\begin{abstract}
1 ARNA - UMR5320 CNRS - U1212 INSERM, Université de Bordeaux, IECB, F-33600
Pessac,

*To whom correspondence should be addressed:

IECB, 2 rue Robert Escarpit, 33607 Pessac - France, E-mails: m.sissler@iecb.u-bordeaux.fr, yaser.hashem@inserm.fr
\end{abstract}

The folding of the ribosomal RNAs is central to the biogenesis of the mitoribosome, a complex, stepwise process. Five recent cryo-EM studies detail the late steps of the folding and maturation of the human mitoribosomal large subunit RNA forming the catalytic core of the ribosome: the Peptidyl Transferase Center (PTC).

The human mitochondrial ribosome (mitoribosome) synthesizes a subset of 13 proteins that are all subunits of respiratory chain complexes and thus are crucial for the synthesis of cellular ATP ${ }^{1}$. Still, very little is known about the regulation of the mitoribosome biogenesis and activity. The biogenesis of the cytosolic eukaryotic ribosomes has been extensively studied in the past few years ${ }^{2,3}$ and its understanding made remarkable advances thanks to numerous structural investigations by cryo-electron microscopy (cryo-EM). However, these findings cannot be fully extended to the animal mitochondrial ribosomes ${ }^{4}$. Cipullo et al. ${ }^{5}$, Hillen et al. ${ }^{6}$, Cheng et al. ${ }^{7}$, Lenarčič et al. ${ }^{8}$, and Chandrasekaran et al. ${ }^{9}$ independently investigate the late steps of the maturation of the human large mitoribosomal subunit (LSU), using different sample preparation and purification techniques, but all relying on cryo-EM for the structure resolution. More specifically, these studies focus on the investigation of the PTC that is made exclusively of rRNA forming the ribosomal device responsible for the polymerization reaction of the incoming amino acids into protein chains.

The biogenesis of the mitoribosome is complex and peculiar for two main reasons. First, the rRNAs (and the tRNA that acts as 5S rRNA) are encoded by the mitochondrial genome, and are thus transcribed and processed inside the mitochondria, while all the other constituents (ribosomal proteins and maturation factors) are encoded by the nuclear genome and imported into the mitochondria subsequently to their synthesis in the cytosol ${ }^{1}$. Second, mammalian mitoribosomes have undergone a reductive phase of rRNA evolution, where rRNAs are substantially reduced when compared to their bacterial ancestors and to plant mitoribosomes 10,11, and where novel ribosomal proteins were acquired (constructive evolution of proteins) ${ }^{12}$. It is believed that the mitochondria of highly active organisms, including mammals, produce large amounts of reactive oxygen species (ROS), creating a challenging environment for the ribosome to function. This in turn could explain why mammalian rRNAs, in addition to being of reduced size, are significantly deprived of guanine residues, which are the most easily oxidized nucleic acid bases ${ }^{13}$. Thus, many regions of 
the mitoribosome have undergone substantial rearrangements to adapt to the unique mitochondrial environment ${ }^{13}$. In spite of the strong evolution pressure towards reducing the rRNAs in mammals, many mitoribosomal features maintained their RNA nature and are strictly conserved, such as the PTC, which is largely inherent to the catalytic capabilities of RNA.

Cipullo et al. ${ }^{5}$, Hillen et al. ${ }^{6}$, Cheng et al. ${ }^{7}$, Lenarčič et al. ${ }^{8}$, and Chandrasekaran et al. all report different structures of the LSU during maturation at the late stages of the process, but all are centered around the PTC. In all the reported structures, several maturation factors can be found bound at different regions of the maturating LSU. For simplicity sake, we will focus exclusively on these factors, excluding the interplay with any mitoribosomal proteins. Figure 1 summarizes these maturation factors, along with their presumed functions and alternative names. Some of these factors are detected in all five studies, as hallmarks of the captured stage of the PTC maturation. Namely, NOP2/Sun RNA methyltransferase 4 (NSUN4), forming a heterodimer with the mitochondrial transcription termination factor 4 (mTERF4), along with the anti-association triptych MALSU1·LOR8F8・mt-ACP (ACP module). Very interestingly, several GTPases (GTPBP 5, 6, 7 and 10) were found to play important mechanical roles linked to probing the state and remodeling of the target rRNA helices. However, different GTPases can be found in different structures. Finally, modification enzymes related to the methylation of specific rRNA bases (MRM2 and MRM3) can also be found in three of the five studies ${ }^{5,7,8}$.

Although all studies describe the same stage of the LSU maturation, each study unravels distinct details and intermediate complexes. Put together, the structures derived from the five studies can recapitulate the maturation of the PTC (Figure 2). The process starts at a state where domains I, II, III and VI of the rRNA (the peripheral rRNA domains) are already folded and surrounded by most of the mitoribosomal proteins. However, the core rRNA domains IV and $\mathrm{V}$ are only partially folded. At this stage, the ACP module interacts with the pre-LSU below the L7/L12 stalk and hinders any premature association with the small ribosomal subunit (SSU) until the LSU has fully matured. The helicase DDX28 interacts with and disentangles domain $\mathrm{V}$ rRNA (mainly helices 80 to 88) while MRM3 (a methyl transferase) methylates G3040 in helix 92 (also called the A-loop), thanks to GTPBP10 that maintains helices 89 to 93 unfolded and stabilized in the appropriate conformation. These details are reported exclusively by Cheng et al. ${ }^{7}$. At this stage helices 81 to 88 are folded correctly and NSUN4/mTERF4 heterodimer binds to domain V rRNA while DDX28 and MRM3 depart from the pre-LSU. Interestingly, although NSUN4 is an rRNA modification enzyme (known to methylate residue C841 in 12S rRNA from the SSU), it appears not involved in any chemical modification process during LSU maturation: Chandrasekaran et al. report the absence of cytosine methylation in the proximity to the NSUN4 binding site in the LSU rRNA 9 . NSUN4/mTER4 interacts directly with domain IV rRNA helices 68 to 71, keeping them unfolded while it exposes the A-loop to MRM2. GTPBP10 departs and MRM2 binds its methylation target (U3039) in helix 92 of the A-loop ${ }^{5,8}$. A third methylation also takes place in helix 80 (also called the P-loop) at residue G2815, however, none of the aforementioned structures capture this transient complex. After the second methylation of the A-loop, GTPBP5 binds the pre-LSU and pushes away the A-loop from MRM2 active site and MRM2 dissociates from the complex ${ }^{5,6,8}$. At this point, GTPBP5 departs and GTPBP7 interacts with the LSU between the ACP module and mTERF4 and acts as a checkpoint for the U3039 methylation ${ }^{9}$.However, Cipullo et al. ${ }^{5}$ finds GTPBP7 bound to a slightly different site (adjacent to MRM2) in a different fashion to that observed by Chandrasekaran et al. ${ }^{9}$, and they suggest its role in monitoring the state of helices 68 to 71 from the domain IV and preventing early SSU joining. Another notable detail reported by the Cipullo et al. is the 
presence of the elongation factor EF-Tu bound to GTPBP5, perhaps underlying the recruitment of the latter to its correct binding site protruding towards the future PTC ${ }^{5}$. Finally, NSUN4/mTERF4 and GTPBP7 leave the pre-LSU and GTPBP6 binds helices 68 to 71 and operates local conformational rearrangements required for their full maturation ${ }^{6}$. The domain V rRNA is thus fully matured. The ACP module departs along with GTPBP6, the PTC is now complete and the LSU is ready to join the SSU and commence translation.

The complementarity between the five different studies sheds light at the PTC maturation with unprecedented details, demonstrating the finesse of the regulation of this process in a great number of consecutive steps. This sequentiality is in part assured thanks to the GTPases involved in the process, which often have mutually exclusive binding sites. It is plausible to view part of their roles as checkpoints where they are able to probe the rRNA, stabilize it and prevent the premature beginning of the next maturation step. The conservation of most of these maturation factors across species (e.g., DDX28 and MRM3 correspond to mt-LAF2 and mt-LAF5/6 in kinetoplastids, respectively ${ }^{14-16}$ ) strongly suggests that the mechanism of the LSU rRNA maturation is conserved. While the maturation of rRNA domains IV and V (the PTC) appears to be a strictly regulated stepwise process, the maturation of the other peripheral rRNA domains may not require this strict sequentiality. Indeed, the analysis of three studies reporting the maturation of the LSU rRNA from the kinetoplastids mitoribosome ${ }^{14-16}$ (from Trypanosoma brucei and Leishmania tarentolae) indicates that the order of different domain II helices folding can vary significantly.

The conservation of the late mitoribosome biogenesis across species suggests similar conservation of the earlier stages of the process and thus can expand our knowledge of the latter. However, it also raises the essential question of nomenclature. Many maturation factors have various alternative names that are often related to the studied organisms, which complicates the understanding of their roles and the establishment of comprehensive models for the mitoribosome biogenesis. We hope that the community will come together to work on a new universal nomenclature that can better reflect the conservation of the mitoribosome maturation factors across species.

Competing interests

The authors declare no competing interests.

1. Hällberg, B.M. \& Larsson, N.-G. Making Proteins in the Powerhouse. Cell Metabolism 20, 226-240 (2014).

2. Klinge, S. \& Woolford, J.L. Ribosome assembly coming into focus. Nature Reviews Molecular Cell Biology 20, 116-131 (2018).

3. Kargas, V. et al. Mechanism of completion of peptidyltransferase centre assembly in eukaryotes. eLife 8(2019).

4. Brown, A. et al. Structures of the human mitochondrial ribosome in native states of assembly. Nature Structural \& Molecular Biology 24, 866-869 (2017).

5. Cipullo, M., Gesé, G.V., Khawaja, A., Hällberg, B.M. \& Rorbach, J. Structural basis for late maturation steps of the human mitoribosomal large subunit. Nature Communications 12(2021).

6. Hillen, H.S. et al. Structural basis of GTPase-mediated mitochondrial ribosome biogenesis and recycling. Nature Communications 12(2021). 
7. Cheng, J., Berninghausen, O. \& Beckmann, R. A distinct assembly pathway of the human 39S late pre-mitoribosome. bioRxiv (2021).

8. Lenarčič, T. et al. Stepwise maturation of the peptidyl transferase region of human mitoribosomes. Nature Communications 12(2021).

9. Chandrasekaran, V. et al. Visualising formation of the ribosomal active site in mitochondria. bioRxiv (2021).

10. Waltz, F. \& Giegé, P. Striking Diversity of Mitochondria-Specific Translation Processes across Eukaryotes. Trends in Biochemical Sciences 45, 149-162 (2020).

11. Waltz, F., Soufari, H., Bochler, A., Giegé, P. \& Hashem, Y. Cryo-EM structure of the RNA-rich plant mitochondrial ribosome. Nature Plants 6, 377-383 (2020).

12. Bieri, P., Greber, B.J. \& Ban, N. High-resolution structures of mitochondrial ribosomes and their functional implications. Current Opinion in Structural Biology 49, 44-53 (2018).

13. Hosseini, M. et al. How to fold and protect mitochondrial ribosomal RNA with fewer guanines. Nucleic Acids Research (2018).

14. Jaskolowski, M. et al. Structural Insights into the Mechanism of Mitoribosomal Large Subunit Biogenesis. Mol Cell 79, 629-644 (2020).

15. Soufari, $\mathrm{H}$. et al. Structure of the mature kinetoplastids mitoribosome and insights into its large subunit biogenesis. PNAS 117, 29851-29861 (2020).

16. Tobiasson, $\mathrm{V}$. et al. Interconnected assembly factors regulate the biogenesis of mitoribosomal large subunit. The EMBO Journal 40(2021).

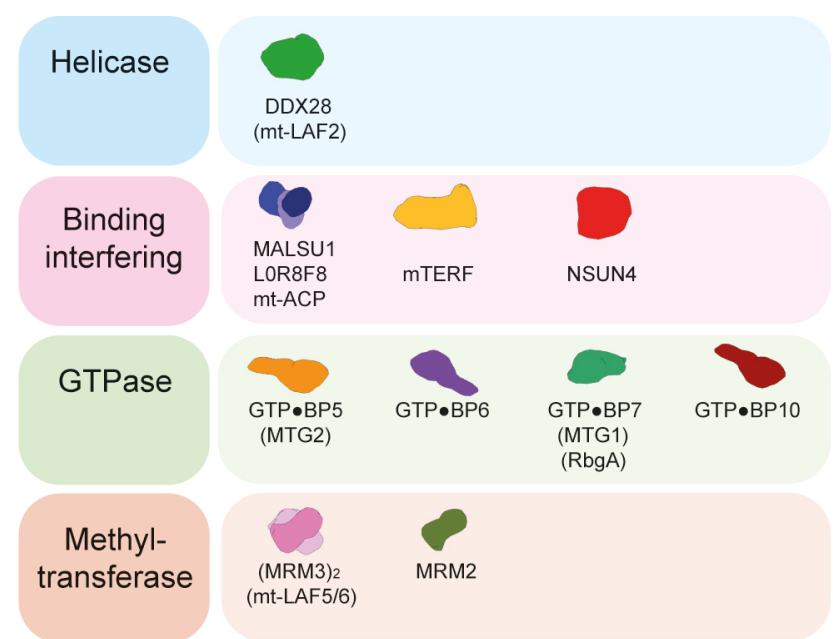

Figure 1. Mitoribosomal maturation factors. Structurally captured human mitoribosomal maturation factors that participate in the late-stage maturation of the large mitoribosomal subunit. Some of these factors are known under alternative names (shown in brackets). The functions of the maturation factors are indicated in the left column. 


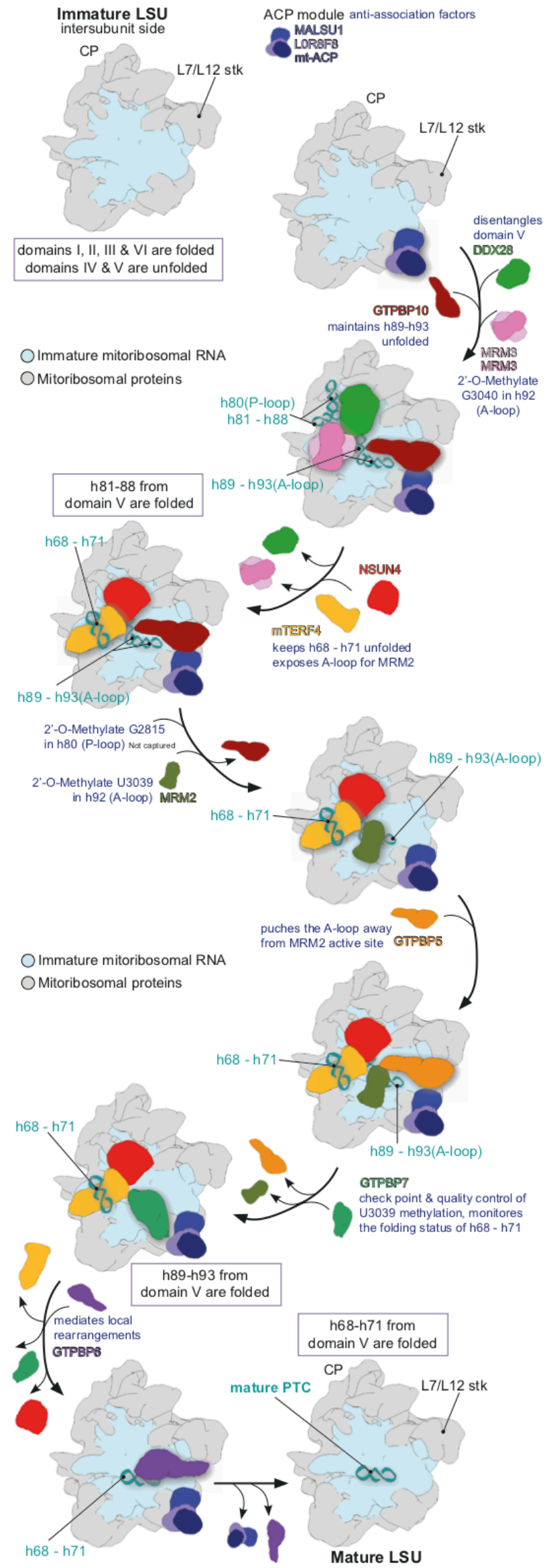

Figure 2. Synthetic model of the latestage large mitoribosomal subunit maturation process. The model recapitulates the different maturation snapshots revealed by the five highlighted studies. The status of the intermediate complexes is indicated inside purple boxes. The function of each maturation factor is indicated in blue under its name. Immature rRNA elements are presented as helices in dark cyan ribbons. Once an rRNA element has matured, it is removed from the scheme sequentially. The sequence of interactions was deduced from the compilation of the five highlighted studies. 